Draft Version November 2, 2018

Preprint typeset using $\mathrm{L}^{A} \mathrm{~T}_{\mathrm{E}} \mathrm{X}$ style emulateapj v. 08/22/09

\title{
OBSERVATIONAL SIGNATURES OF SIMULATED RECONNECTION EVENTS IN THE SOLAR CHROMOSPHERE AND TRANSITION REGION
}

L. HegGland

Institute of Theoretical Astrophysics, University of Oslo, P.O. Box 1029, Blindern, N-0315 Oslo, Norway

B. De Pontieu

Lockheed Martin Solar and Astrophysics Laboratory, 3251 Hanover Street, Org. ADBS, Building 252, Palo Alto, CA 94304

\author{
V. H. Hansteen ${ }^{1}$ \\ Institute of Theoretical Astrophysics, University of Oslo, P.O. Box 1029, Blindern, N-0315 Oslo, Norway \\ Draft version November 2, 2018
}

\begin{abstract}
We present the results of numerical simulations of wave-induced magnetic reconnection in a model of the solar atmosphere. In the magnetic field geometry we study in this article, the waves, driven by a monochromatic piston and a driver taken from Hinode observations, induce periodic reconnection of the magnetic field, and this reconnection appears to help drive long-period chromospheric jets. By synthesizing observations for a variety of wavelengths that are sensitive to a wide range of temperatures, we shed light on the often confusing relationship between the plethora of jet-like phenomena in the solar atmosphere, e.g., explosive events, spicules, blinkers, and other phenomena thought to be caused by reconnection.
\end{abstract}

Subject headings: magnetic fields - MHD — Sun: chromosphere — Sun: transition region

\section{INTRODUCTION}

The Sun displays a bewildering array of jet-like phenomena that can be observed at many different wavelengths and in many different regions. At the limb, we find spicules, protrusions of cool gas that can reach heights of $6-10 \mathrm{Mm}$ and have lifetimes of several minutes (e.g. Beckers 1968), and that are observed in chromospheric lines such as $\mathrm{H} \alpha$ and $\mathrm{Ca}$ II $\mathrm{H}$. There are also larger and longer-lasting jets such as surges and macrospicules. Recently, faster and shorter-lived jets, called type II spicules, have been found (De Pontieu et al. 2007b), many lasting less than $100 \mathrm{~s}$ before fading from view. Hinode observations were instrumental in finding these, but have also shown a large amount of other jet activity in $\mathrm{Ca} \mathrm{H}$ and other lines (e.g. Katsukawa et al. 2007; Shibata et al. 2007).

On the disk, mottles show much of the same behaviour as spicules, and we can also observe shorter-lived dynamic fibrils in the same lines. In higher-temperature ultraviolet lines such as C IV, Si IV, and O VI, we observe short-lived explosive events and longer-lasting blinkers. The former, in particular, have very wide line profiles indicating strong bidirectional jets (Innes et al. 1997).

There have been investigations into whether some of these terms simply describe different aspects of the same basic phenomena, e.g. spicules and mottles (Grossmann-Doerth \& Schmidt 1992; Tsiropoula et al. 1994), blinkers and explosive events (Chae et al. 2000), spicules and explosive events (Wilhelm 2000), or spicules, blinkers, and explosive events (Madiarska \& Dovle 2003). Different authors often conclude differently, and

\footnotetext{
Electronic address: lars.heggland@astro.uio.no

1 Also at Center of Mathematics for Applications, University of Oslo, P.O. Box 1053, Blindern, N-0316 Oslo, Norway
}

the matter can not be considered settled. Part of the problem is that we usually lack cotemporal, cospatial observations across several wavelength bands, so that we most often cannot trace events across different temperatures and with enough spatial and temporal resolution to elucidate the relationship between the various features. Another complication is that we cannot observe the exact same phenomenon both on the disk and at the limb, for simple geometric reasons. Simulations can be very helpful here in letting us change our vantage point at will.

The driving mechanism of the various jets is in general not well established. The main candidates have been waves (magnetoacoustic or Alfvén) and magnetic reconnection. There are strong indications that acoustic shockwaves cause dynamic fibrils (Hansteen et al. 2006; De Pontieu et al. 2007a; Heggland et al. 2007) and that reconnection causes explosive events (Innes et al. 1997; Chae et al. 1998), but the case is more open for the other types of jets. It has been noted by e.g. Chae et al. (1998), Ning et al. (2004), and Dovle et al. (2006) that many explosive events occur in bursts, often with intervals of 3-5 minutes. These periods correspond to the dominant wave modes produced by the solar granulation, suggesting that such waves may induce or modulate reconnection events.

A review of various spicule models can be found in Sterling (2000). Most models so far are in 1D, assuming a rigid magnetic flux tube either with or without expansion. Some use a piston driver, either at a given frequency or randomised, while others use a sudden increase in pressure and temperature as a trigger mechanism for setting up shocks. This pressure increase is often assumed to be in the photosphere, but Shibata et al. (1982) and Sterling et al. (1993) have investigated the ef- 
fects of energy input in the upper chromosphere as well, producing different types of jets that have similar properties to spicules or surges. The source of the energy input is usually not specified, though reconnection could be a natural candidate, as speculated by Sterling et al. (1993).

2D simulations containing an actual magnetic field (as opposed to treating it merely as a given rigid flux tube) have been performed by Takeuchi \& Shibata (2001a,b). They studied photospheric reconnection which they claim produces a large enough wave energy flux to drive spicules, but their actual simulation box does not extend past the lower chromosphere.

Explosive events have been modelled in 1D by Erdélyi et al. (1999) and Sarro et al. (1999). 2D simulations of explosive events have been performed by Karpen et al. (1995), Innes \& Tóth (1999), Roussev et al. (2001a,b, c), and Chen \& Priest (2006). Again, the 1D simulations generally assume a rigid flux tube geometry, and treat the magnetic reconnection not explicitly, but as a sudden deposition of energy at a specified height. The $2 \mathrm{D}$ simulations often assume a simplified magnetic geometry with vertical antiparallel field lines. Karpen et al. (1995) use a more complicated magnetic field geometry with some similarities to the one used in this paper, but do not include radiative losses or heat conduction in their simulations.

In this paper, we present the results of $2 \mathrm{D}$ simulations that include the effects of radiation and heat conduction, and that involve a complex magnetic field geometry in which reconnection events are induced by waves propagating upwards from the photosphere/convection zone. We do not attempt to match the results to specific observations, but show the signatures of these events in several chromospheric, transition region and lower coronal spectral lines, and viewed both on the disk and at the limb of the Sun.

\section{SIMULATIONS}

The model atmosphere is similar to the one used by Heggland et al. (2007), but now the lower boundary of the domain reaches down to the upper photosphere, as opposed to the chromosphere. The simulation box extends about $11 \mathrm{Mm}$ in height, going from the photosphere through the chromosphere, transition region and lower corona. The lower boundary, $z=0$, corresponds to a height of $150 \mathrm{~km}$ in the VAL3C model (Vernazza et al. 1981). The simulation box contains $201 \times 191$ grid cells, using a uniform spacing of $50 \mathrm{~km}$ in the horizontal direction and a non-uniform spacing in the vertical direction, starting at $16 \mathrm{~km}$ in the chromosphere and transition region and increasing exponentially in the corona, reaching $220 \mathrm{~km}$ at the upper boundary. In Figure 1, we have plotted the initial temperature distribution with magnetic field lines and curves of equal plasma $\beta$, the latter defined as the ratio between the thermal and the magnetic pressure.

The magnetic field configuration is a potential field, with a central flux tube which progressively widens with height and ends up dominating the field in the corona. However, there are also two inclined flux tubes, one on each side of the centre. These tubes both end up in magnetic null points, located at transition region heights $(z=1.75 \mathrm{Mm}, x=2.6 \mathrm{Mm}$ and $x=7.4 \mathrm{Mm})$. The null

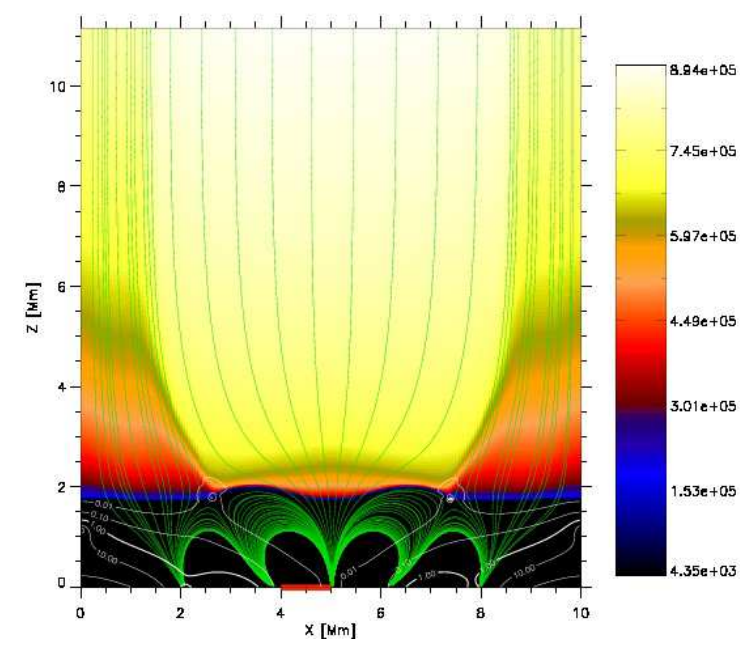

FIG. 1.- Initial temperature structure, with overplotted $\beta$ contours (white) and magnetic field lines (green). The red line between $x=4$ and $x=5 \mathrm{Mm}$ marks the location of the piston. The magnetic null points show up as local maxima of the $\beta$ at $z=1.75 \mathrm{Mm}$ and $x=\{2.6,7.4\} \mathrm{Mm}$.

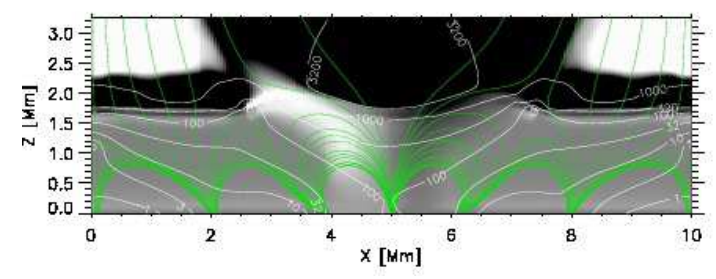

FIG. 2.- Plot of the vertical velocity, set to saturate at $1 \mathrm{~km} \mathrm{~s}^{-1}$. Selected magnetic field lines in green, Alfvén speed contours in $\mathrm{km} \mathrm{s}^{-1}$ in white. The first fast mode wave reaches the transition region after 12 seconds, refracts away from the high Alfvén speed in the centre, and perturbs the field in the vicinity of the magnetic null points, triggering reconnection.

points can be seen in Figure 1]as $\beta$ maxima that are very small in spatial extent. It is near these null points that the reconnection events we study in this paper occur. Finally, there are two outer flux tubes that open into the corona.

It should be noted that this null point configuration is somewhat peculiar to the $2 \mathrm{D}$ geometry, and would be difficult to reproduce exactly in a 3D model, or indeed on the real Sun. However, it is our belief that the observational signatures of reconnection events on the real Sun should not be strongly dependent on the specific geometry of the magnetic field, and that our simulated observations should be broadly similar to what we would expect to see when observing actual reconnection events. This is supported by the similarities we find with observations of several types of events thought to be caused by reconnection.

The code we use is the same 3D MHD code that was used in Heggland et al. (2007), and is described in more detail by Hansteen (2005). It should be noted that it contains a realistic radiative loss function based on collisional excitation of hydrogen, carbon, oxygen, neon, and iron, as well as thermal conduction along the magnetic field (see Heggland et al. (2007) for the details).

In the models presented here we operate with a mag- 
netic diffusivity $\eta$ many orders of magnitude larger than that on the Sun, and dissipation starts at much smaller magnetic field gradients. The dissipated energy is

$$
Q_{\mathrm{Joule}}=\mathbf{E} \cdot \mathbf{J}
$$

where $\mathbf{J}=\nabla \times \mathbf{B}$ is the current density and the resistive part of the electric field is given by

$$
E_{x}^{\eta}=\left\{\frac{1}{2}\left(\eta_{y}^{(1)}+\eta_{z}^{(1)}\right)+\frac{1}{2}\left(\eta_{y}^{(2)}+\eta_{z}^{(2)}\right)\right\} J_{x}
$$

and similar for $E_{y}$ and $E_{z}$. The diffusivities are given by

$$
\begin{aligned}
\eta_{j}^{(1)} & =\frac{\Delta x_{j}}{\operatorname{Pr}_{M}}\left(v_{1} c_{f}+v_{2}\left|u_{j}\right|\right) \\
\eta_{j}^{(2)} & =\frac{\Delta x_{j}^{2}}{\operatorname{Pr}_{M}} v_{3}\left|\nabla_{\perp} \cdot \mathbf{u}\right|_{-}
\end{aligned}
$$

where $\operatorname{Pr}_{M}$ is the magnetic Prandtl number, $c_{f}$ is the fast mode speed, $v_{1}, v_{2}$ and $v_{3}$ are dimensionless numbers of order unity and the other symbols retain their usual meanings.

The upper coronal boundary is maintained at $1 \mathrm{MK}$, while radiation and heat conduction set the temperature structure in the rest of the domain.

In the simulations, we investigate the effects of different locations and methods of driving, and how the produced waves can trigger reconnection. In one case, we use a monochromatic localised piston with $300 \mathrm{~s}$ period and $1.1 \mathrm{~km} \mathrm{~s}^{-1}$ amplitude, driving the lower boundary in the vertical direction at $z=0 \mathrm{Mm}$ between $x=4 \mathrm{Mm}$ and $x=5 \mathrm{Mm}$ (marked with a red line in Fig.11). In another, we use a driver taken from observations with Hinode/SP (Lites, private communication), producing waves at all locations along the lower boundary and in a more realistic range of periods. The data were obtained on 2007 October 22 and contain the line-of-sight velocities in a slice across a network element.

We also experiment with putting the null points at different heights in the atmosphere. In one case, they are located in the transition region at a height of about 1.75 Mm above the lower boundary, as in Figure1, in another, they are in the upper chromosphere, some $450 \mathrm{~km}$ lower.

\section{ANALYSIS}

\subsection{Transition region null points, piston driver}

\subsubsection{General description of events}

We first look at the case with null points in the transition region and the localised piston driver as described above. Due to the varying magnetic inclination in the region of the piston, it will generate a mixture of fast and slow mode waves. In low- $\beta$ regions, which include the piston location as well as most of the simulation box, the fast modes can propagate everywhere, though they tend to be refracted into regions of low Alfvén velocity (e.g. Osterbrock 1961), such as null points. The slow modes are mainly restricted to propagation along the magnetic field.

The waves disturb the field as they travel, and once the disturbance reaches the null points, reconnection is triggered as lines of opposite polarity are pushed together and meet. This process releases a significant amount of energy in a short time and leads to very rapid heating of the plasma; in the stronger events, the plasma around the null points reaches coronal temperatures of roughly $1 \mathrm{MK}$. In addition, a bidirectional jet is formed, as plasma is rapidly accelerated away from the reconnecting region.

Since the lines of opposing polarity are located quite close to each other around the null point, only a small disturbance is required to start off the reconnection process the first time. This happens soon after the start of the simulation, as fast mode waves generated by the piston in the central flux tube propagate upwards and reach the transition region in as little as 10-12 seconds. There, they are refracted away from the high Alfvén speed region in the centre of the box (see Fig. 2) and move instead towards the sides, where they reach the magnetic null points and push the field lines together, triggering reconnection.

The piston, driving generally along the field in a magnetically dominated (i.e. low- $\beta$ ) plasma, is more efficient at generating slow mode than fast mode waves, so these initial fast disturbances have low amplitude - around $1 \mathrm{~km} \mathrm{~s}^{-1}$ just before reaching the transition region but this is enough to push the field lines above and below the null point into each other and trigger a weak reconnection event. This leads to moderate heating of the reconnecting region and the formation of a bidirectional jet, with high velocities upwards to the left and downwards to the right. The hot jet going left then pushes cooler material sideways and upwards, propelling a small, spicule-like protrusion of cooler chromospheric material into the corona (Fig. 3, left panel).

After some 160 seconds, or half a driver period later, the piston driven disturbance changes sign, and starts pulling the field lines above and below the null point away from each other. Instead, the lines to the left and right of the null point are pushed together, and reconnection occurs again, this time in a direction perpendicular to the original reconnection event. Another bidirectional jet is formed, this time being mostly vertical, and the region around the null point (now embedded entirely in cooler chromospheric gas) is heated to coronal temperatures. This heated region spreads out along the jets and the magnetic field, and creates a butterfly-shaped region of heated plasma (Figure 3, left centre panel).

The orientation of the upwards-moving jet changes as the reconnection continues, and it rotates from being directed upwards to the right (mainly across the field lines) to being directed somewhat to the left (mainly along the field lines). The latter orientation allows the gas to move much more freely, and the hot plasma is soon accelerated upwards along the field lines with great speed (Fig. 3, right center panel). As it passes the now mainly sideways moving cool jet generated by the first reconnection, it pushes some of that cool gas upwards as well and greatly extends the length of the cool jet, which reaches a maximum height of $z=8 \mathrm{Mm}$.

This pattern then repeats itself, with reconnection being triggered every 150 s (i.e. every half period), alternating between vertical and horizontal orientation, and propelling both cool and hot jets upwards. The later events are not as strong as the first one, and are quite similar to each other. An example of a later horizontally oriented reconnection jet is shown in the right panel of 

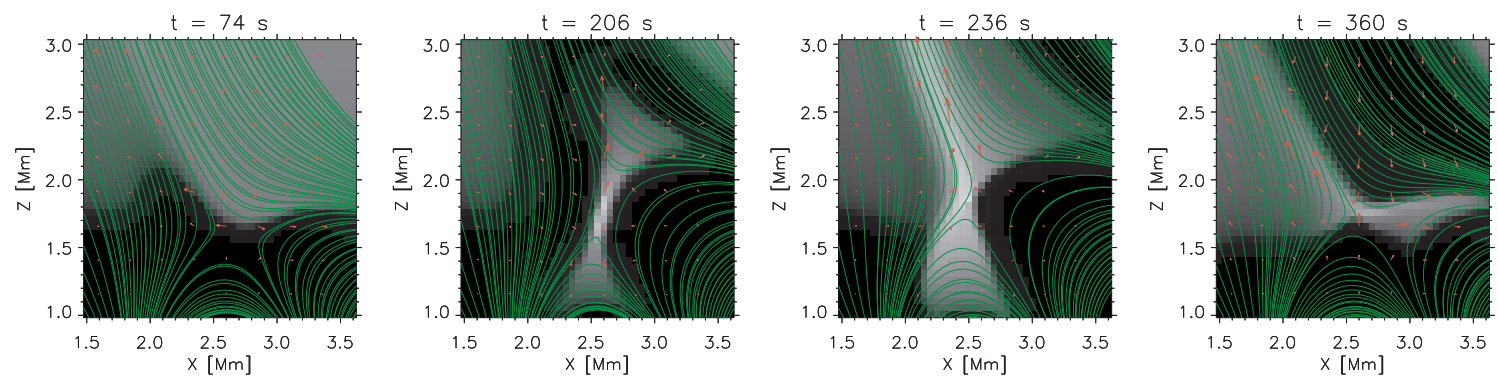

FIG. 3.- Plots of the plasma temperature (greyscale) with magnetic field lines (green) and velocity vectors (red) at four different times in the model with transition region null points and piston driver. The plots zoom in on the area around the left-hand null point. At $74 \mathrm{~s}$ (left panel), a horizontal bidirectional jet is pushing cool material upwards to the left. At $206 \mathrm{~s}$, reconnection has heated a significant area and propels a jet mainly across the field lines. At $236 \mathrm{~s}$, the jet aligns more with the field and hot material is ejected upwards. The final panel shows a later horizontally oriented reconnection after $360 \mathrm{~s}$. The field lines in all panels are drawn to show the geometry rather than the strength of the magnetic field.

Figure 3 .

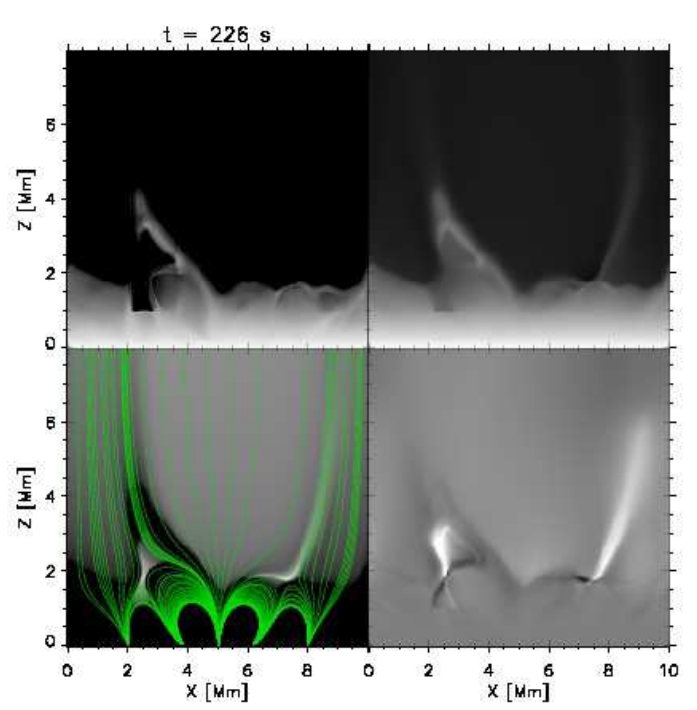

FIG. 4.- Plots of the Ca signal (top left), density (top right), temperature with magnetic field lines (bottom left), and field-aligned velocity (bottom right) in the case with transition region null points and piston driver. The elapsed time is $226 \mathrm{~s}$. Reconnection is producing a butterfly-shaped, vertically oriented heated region and jet on the left, and a hot TR jet on the right. This figure is available as a movie in the electronic version of the Journal.

\subsubsection{Synthesised limb observations}

In order to get an impression of how these jets would look if observed at the solar limb, we have used the nonLTE radiative transfer code MULTI (Carlsson 1986) to calculate the population densities of the different excitation levels of Ca II ions in our simulations. In the side views, we have chosen to concentrate on the $\mathrm{Ca}$ II $\mathrm{H}$ line at $3968 \AA$. In a $2 \mathrm{D}$ model, it is not possible to do a full radiative transfer calculation along a line perpendicular to the plane of the computational box, but the population density of the upper level of this transition serves as a rough approximation of the actual intensity.

In Figure 4, we show plots of the logarithm of the calculated Ca II H upper level density (upper left, henceforth and in the figure captions referred to as the Ca signal), the logarithm of the mass density from the MHD simulation data (upper right), the plasma temperature with overplotted magnetic field lines (lower left), and the velocity along the magnetic field (lower right) after $226 \mathrm{~s}$. 


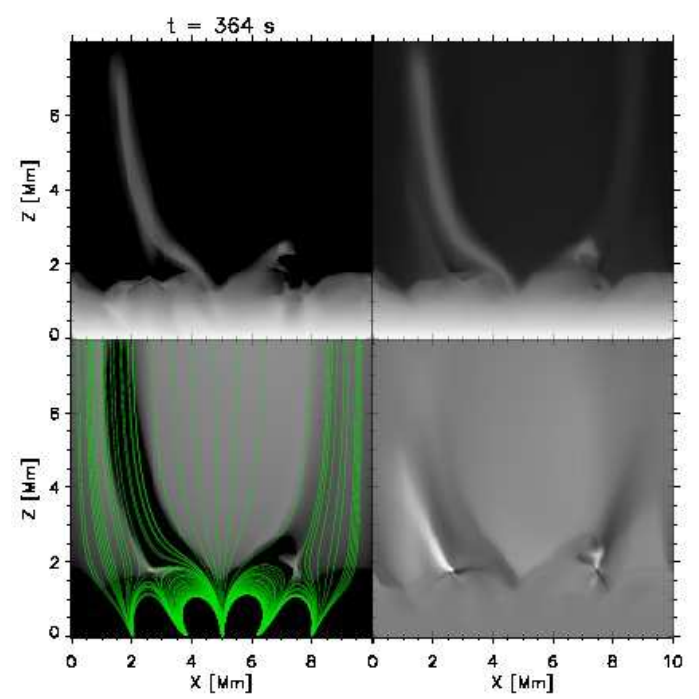

FIG. 5.- Plots of the Ca signal (top left), density (top right), temperature with magnetic field lines (bottom left), and field-aligned velocity (bottom right) in the case with transition region null points and piston driver. The elapsed time is $364 \mathrm{~s}$. The spicule-like cool jet on the left has reached its greatest length, extending nearly $8 \mathrm{Mm}$ above the photosphere.

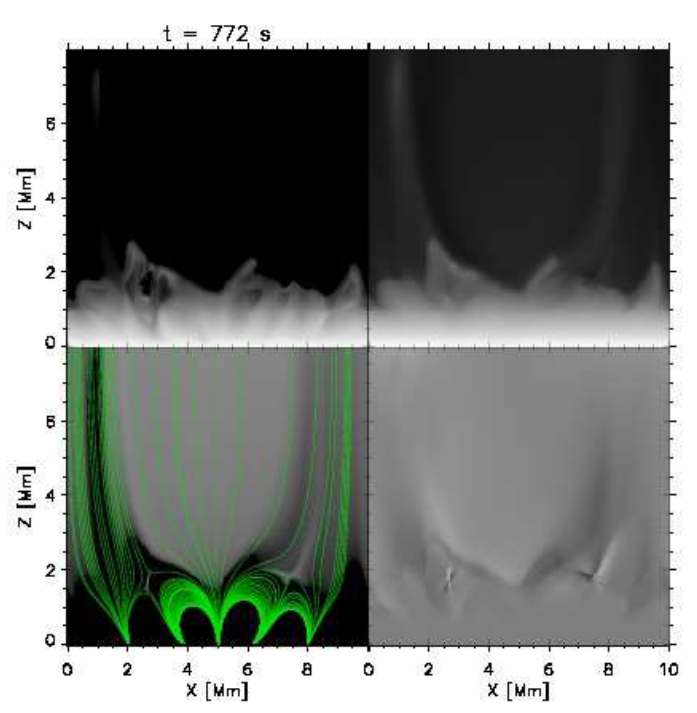

FIG. 6.- Plots of the Ca signal (top left), density (top right), temperature with magnetic field lines (bottom left), and field-aligned velocity (bottom right) in the case with transition region null points and piston driver. The elapsed time is $772 \mathrm{~s}$. A thin, low-density cool jet can be seen on the left in the temperature plot.

At this time, reconnection with vertically oriented jets is happening near the left-hand null point, while a horizontally oriented event is happening near the right-hand null point - though the jet on the right-hand side of it is field-aligned and has turned upwards quite sharply. The cooler jets show up in Ca II as rather thin, elongated features, similar to spicules or fibrils. In contrast, the hotter jets, and the reconnection regions themselves, show no Ca II signal. The hot jet on the right does have a density enhancement (upper right panel), but it and the regions around the null points are heated to temper- atures of about $1 \mathrm{MK}$, far above the temperature where calcium gets multiply ionised, which explains the lack of $\mathrm{Ca}$ II emission. The high velocities of the reconnection jets are clearly visible in the lower right panel.

The cooler jets are seen to rise and fall; in Figure 5 we show the situation after $364 \mathrm{~s}$, when the cool jet seen on the left in Figure 4 has reached its maximum extent, after being pushed upwards by the hot jet from the reconnection event at 200-240 s (ref. Fig. 3). It reaches a height of nearly $8 \mathrm{Mm}$.

In Figure 6, we see a later, thinner cool jet on the left, which shows up only weakly in Ca II. The density plot (upper right) tells us why: its density appears to be too low, even though it is in about the right temperature range.

The typical lifetimes of the cool jets are 200-300 s, and they reach maximum lengths of 6-8 Mm above the photosphere. Their thickness varies a lot with time, but is typically slightly less than $1 \mathrm{Mm}$. These figures are roughly within the ranges reported for spicules (Beckers 1968). However, it is important to look at the whole range of spectral, spatial and temporal data available before trying to establish correspondence between simulations and observations.

\subsubsection{Synthesised disk observations}

In producing synthesised disk observations, we look into the simulation box directly from above, in the plane of the box. A proper radiative transfer treatment is then possible, and has been carried out for the Ca II IR line at $8542 \AA$, widely used in spectroscopic studies. The calculations have been made column by column, i.e. neglecting any radiative interaction in the $x$-direction. A simplified treatment based only on collisional excitation has been carried out for the optically thin lines of C IV (1548 $\AA)$, O VI (1032 $\AA)$, and Fe XII (195 $\AA)$. These latter lines will typically be formed in the transition region or lower corona, whereas the Ca IR line is formed in the chromosphere. In the treatment of the Fe line, which has a wavelength shorter than the Lyman threshold at $912 \AA$, we have included the effects of absorption by neutral hydrogen and neutral and singly ionised helium.

$\lambda$-t plots of these lines, looking directly down on the centre of the reconnection region at $x=2.6 \mathrm{Mm}$, are shown in Figure 7 . The $\mathrm{C}, \mathrm{O}$, and Fe lines have had their resolution and cadence downgraded to typical instrumental values of $650 \mathrm{~km}$ (about 1") and $20 \mathrm{~s}$, and are plotted using logarithmic scaling to bring out details in the weaker later events. Ca is plotted at $150 \mathrm{~km}$ resolution and $2 \mathrm{~s}$ cadence, using linear scaling.

In the transition region lines, there is a sudden onset of a very bright, very broad feature, with upflows of 80$90 \mathrm{~km} \mathrm{~s}^{-1}$ and downflows of $60-80 \mathrm{~km} \mathrm{~s}^{-1}$. This happens first in C IV, the lowest temperature line, at $160 \mathrm{~s}$, then in $\mathrm{O}$ VI at $180 \mathrm{~s}$ (with a weaker signal at $160 \mathrm{~s}$ ). This feature is caused by the sudden heating and the powerful bidirectional jet generated by the reconnection. These spectra show strong similarities to observations of explosive events (Innes et al. 1997; Chae et al. 2000), which are believed to be caused by reconnection. Dere (1994) finds that explosive events have typical lifetimes of $60 \mathrm{~s}$, spatial extents of about 1.5 Mm, and typical Doppler shifts of $100 \mathrm{~km} \mathrm{~s}^{-1}$, though both higher and lower dura- 


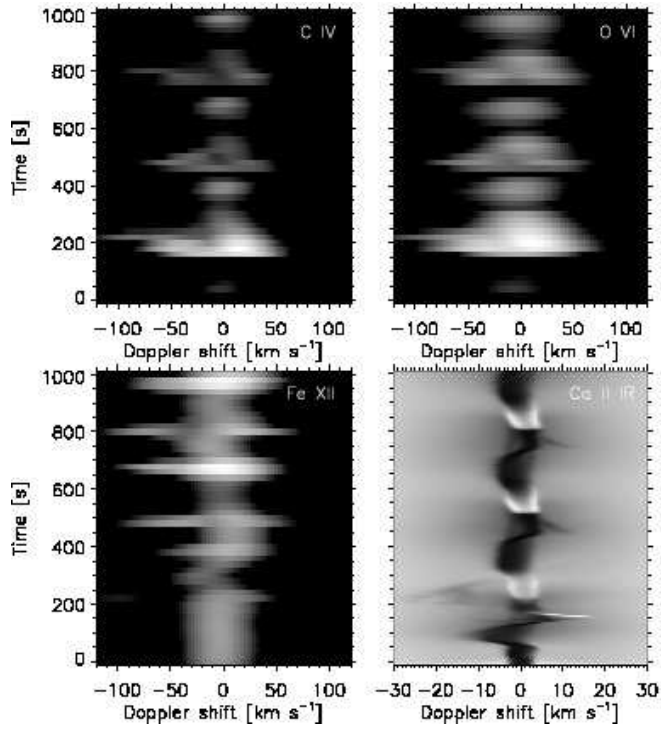

FIG. 7. - Spectra of the high null point piston driver case, centered at $x=2.6 \mathrm{Mm}$ and with blueshifts shown as negative velocity. The $\mathrm{C}$, $\mathrm{O}$ and Fe lines use logarithmic scaling in order to bring out the details of the much weaker later events. The difference between white (maximum intensity) and black (intensity cutoff) is a factor $10^{4}$. The UV lines experience strong brightening and extensive Doppler shifts when the reconnection events happen. The profiles are asymmetric, having higher blueshifts than redshifts, but the intensity maximum is slightly redshifted. The Ca spectrum uses linear scaling and a much smaller Doppler shift scale.

tions and Doppler shifts have been reported. The figures match up well with our results; the main parts of the brightenings fade away after $60 \mathrm{~s}$ and the spatial extent is roughly in the quoted range (see Figs. 3 and 4).

The profiles are asymmetric, with larger blueshifts than redshifts, and in particular the $\mathrm{C}$ and $\mathrm{O}$ lines have a short-lived blueshift of $140-150 \mathrm{~km} \mathrm{~s}^{-1}$ at $220 \mathrm{~s}$. On the other hand, the intensity maximum is redshifted. This behaviour can be readily understood in terms of the density stratification. The reconnection outflow jets are expected to propagate outwards at the Alfvén speed, which is inversely proportional to the square root of the density. Hence, the jets propagating upwards in a stratified medium should be faster than those propagating downwards, leading to an asymmetry like the one we find. The blueshift maximum between 220 and 240 s happens when the heated jet, which is initially embedded in material at chromospheric temperatures (Fig. 4), enters the corona where the Alfvén speed increases markedly. Meanwhile, the compression effect of the jet should be stronger for the part propagating downwards into denser material, leading to higher emission at slight redshifts.

The temperature in the reconnection region gets high enough to produce significant emission also in the Fe XII line, but it is quite strongly affected by neutral hydrogen absorption. The first powerful event is completely absorbed because the reconnection region at that time is located under a small jet with at least $1 \mathrm{Mm}$ of chromospheric material above it. The first horizontal jet reconnection event shows up weakly at around $400 \mathrm{~s}$, but strong blue- and redshifts are not seen until the vertical jet at $500 \mathrm{~s}$. The intensity maximum is in a horizontal jet at around $680 \mathrm{~s}$, at which time there is hardly any chromospheric material above the reconnection region.
The chromospheric Ca II IR line shows signs of activity before any of the higher temperature lines. A relatively strong upflow $\left(15 \mathrm{~km} \mathrm{~s}^{-1}\right)$ is caused after less than $100 \mathrm{~s}$ by the first weak reconnection episode. This upflow is soon reversed, and after $150 \mathrm{~s}$ the first major reconnection happens, changing the line centre from a redshift of $20 \mathrm{~km} \mathrm{~s}^{-1}$ to 0 almost instantly, while also yielding a significant blueshifted component with speeds up to $30 \mathrm{~km} \mathrm{~s}^{-1}$.

After this violent first event, several periodic weaker reconnection events follow. In these, the line only undergoes significant redshifts, with very little evidence of blueshifts. This indicates that the line's main formation height is below the reconnection region. The other lines show periodic weaker brightenings, though still with quite high velocity. In every other event, the main jets are oriented horizontally, leading to smaller Doppler shifts. The intensity decrease is mainly due to reduced density in the reconnection region; the reconnection jets cause a large outflow of material, and because the inflow (amplified by the waves) soon triggers another reconnection event, the area never recovers its initial density.

\subsection{Chromospheric null points, piston driver}

\subsubsection{General description}

These simulations use a piston driver of the same period $(300 \mathrm{~s})$ and amplitude $\left(1.1 \mathrm{~km} \mathrm{~s}^{-1}\right)$ as in the previous section. However, here the magnetic null points are located around $450 \mathrm{~km}$ lower in the atmosphere, putting them in the upper chromosphere rather than in the lower transition region. The basic geometry of the magnetic field is not changed, as we can see in Figure 8, the only difference is a translation of the whole geometry to lower heights.

\subsubsection{Synthesised limb observations}

Figure 8 shows the simulation, viewed from the side, at the time when the first and most powerful reconnection event has reached its maximum, at around $260 \mathrm{~s}$ elapsed time. Once more, we have plotted the Ca H signal ( $u p$ per left), the logarithm of the mass density (upper right), the temperature (lower left) and the field-aligned velocity (lower right). We clearly see the butterfly-shaped region of higher temperature (lower left) and the fast bidirectional jet (lower right), although the latter is now going upwards much faster than downwards due to the plasma being able to more easily propagate along the magnetic field. The beginnings of a jet can be seen in $\mathrm{Ca} \mathrm{H}$ (upper left), although the heated region is too hot (and to some extent too evacuated) to show any Ca signal.

In Figure 9, we show the situation as the cool jet reaches its maximum extent, 90 seconds later. As could be expected, the velocity signal is weak at this point, and the region around the null point is no longer heated to coronal temperatures. The jet shows up clearly in $\mathrm{Ca}$, but reaches a maximum height of only about $4.5 \mathrm{Mm}$, as compared to $8 \mathrm{Mm}$ when the null points are located in the transition region. The general pattern described above repeats itself periodically every 300 s, with progressively slower and shorter jets.

Overall, this case is quite similar to the case with higher null points described in the previous section. The 


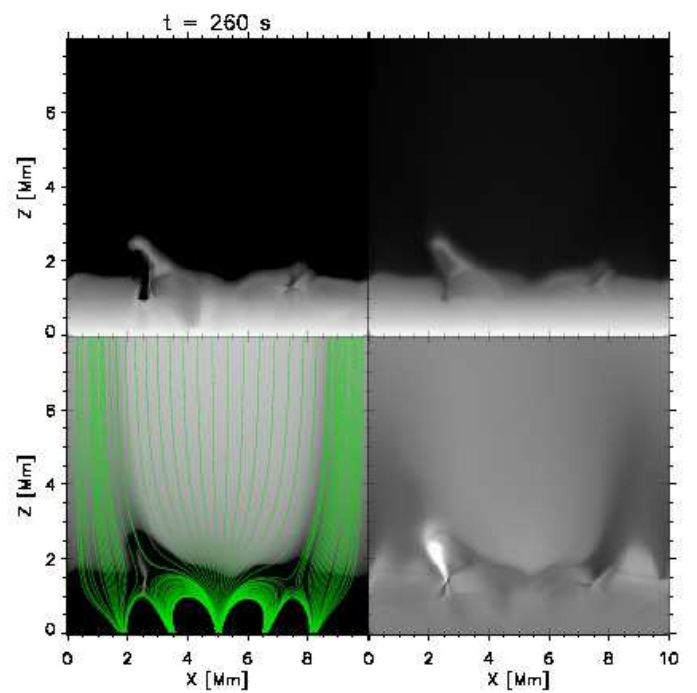

FIG. 8. - Plots of the Ca signal (top left), density (top right), temperature with magnetic field lines (bottom left), and field-aligned velocity (bottom right) in the case with chromospheric null points and piston driver. The elapsed time is $260 \mathrm{~s}$. The outflow speed from the reconnection region on the left is at its maximum. This figure is available as a movie in the electronic version of the Journal.

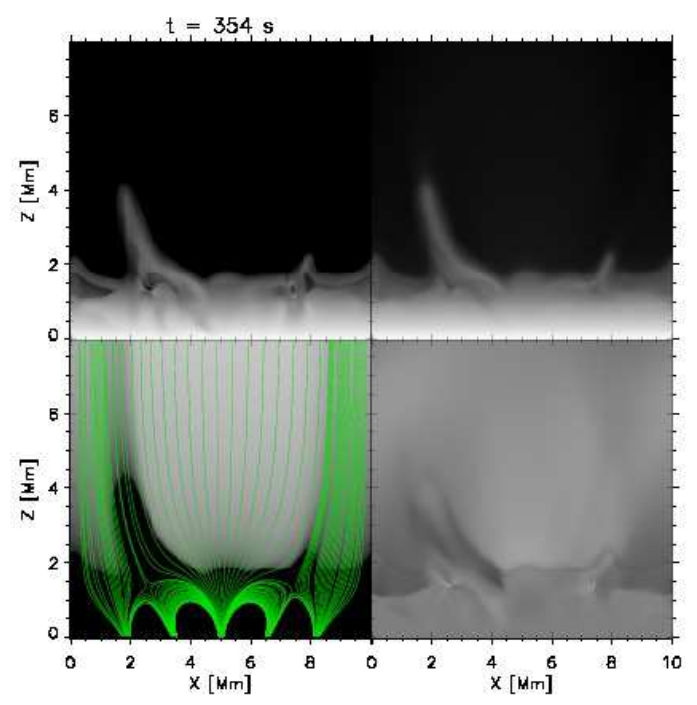

FIG. 9.- Plots of the Ca signal (top left), density (top right), temperature with magnetic field lines (bottom left), and field-aligned velocity (bottom right) in the case with chromospheric null points and piston driver. The elapsed time is $354 \mathrm{~s}$. The extent of the cool jet is at its maximum, $4 \mathrm{Mm}$ above the photosphere.

jets are slower and shorter because of the higher density surrounding the null points, resulting in lower acceleration for similar force.

\subsubsection{Synthesised disk observations}

Figure 10 shows $\lambda$-t diagrams of C IV, O VI, Fe XII and Ca II IR as they would appear looking straight down at the centre of the reconnection region $(x=2.6 \mathrm{Mm})$, as for the high null point case in section 3.1. The resolution is also the same as used previously. In $\mathrm{C}$ IV and $\mathrm{O}$ vI, we get bidirectional signals spaced $300 \mathrm{~s}$ apart,

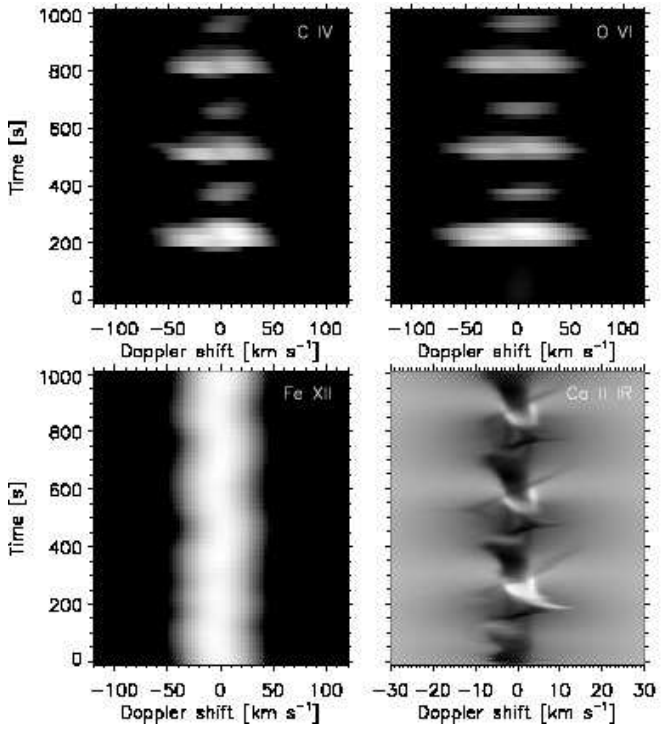

FIG. 10.- Spectra of the chromospheric null point piston driver case, centered at $x=2.6 \mathrm{Mm}$ and using logarithmic scaling for the UV lines and linear scaling for $\mathrm{Ca}$. $\mathrm{C}$ and $\mathrm{O}$ show largely symmetric profiles with maxima on each reconnection event with vertical jets. Fe shows only the background emission. Ca has a complex line profile with multiple components.

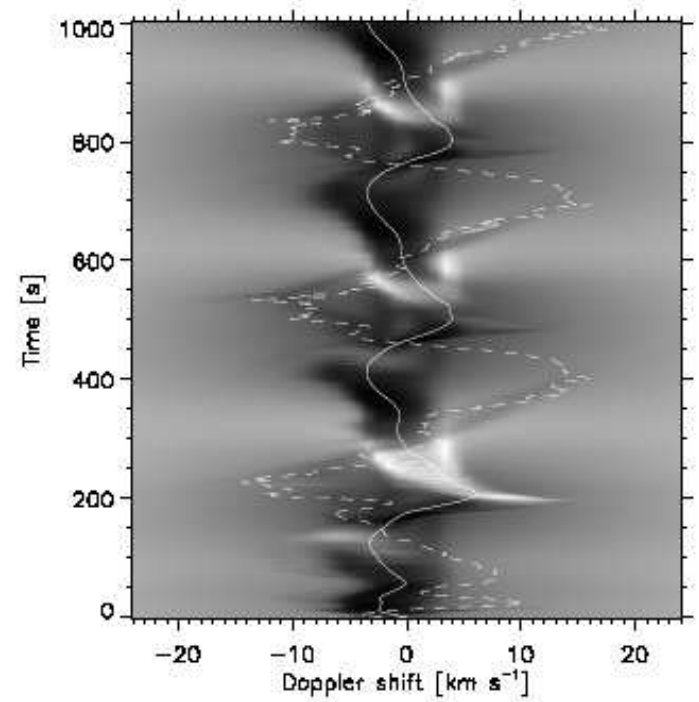

FiG. 11. - Ca spectrum with doppler shifts (greyscale) in the chromospheric null point piston driver case, with velocities at $z=$ $0.93 \mathrm{Mm}$ (solid line) and $z=1.37 \mathrm{Mm}$ (dashed line) superimposed. The former fits well with the line centre position, while the latter is a reasonable match for the blueshifted spikes and their following redshifts.

with Doppler shifts of $40-60 \mathrm{~km} \mathrm{~s}^{-1}$, but little other signal. In Fe XII, the events give no visible signal, and we just see the background emission. The reason for the low $\mathrm{Fe}$ emission is a combination of the relatively lower temperature reached in the region heated by reconnection and greater absorption by neutral hydrogen due to the deeper location of the reconnection region. The UV lines are in general more symmetric in this simulation, because the hot jet does not reach the perturbed transition region and corona before being cooled. 
The Ca line, though, appears highly complex in this simulation. It exhibits both absorption and emission, as well as strong Doppler shifts into both red and blue. In observations, such a spectrum would be difficult to make sense of. However, we have the full simulation data to work with, including the velocity at all heights. In fact, at least two different velocity components can be identified as being responsible for the appearance of the spectrum, as shown in Figure 11. The line centre closely follows the vertical velocity at a height of $0.92 \mathrm{Mm}$, below the magnetic null point, as marked with a solid white line in the figure. The strongest blueshifted signal, which later passes through the line centre and becomes redshifted, corresponds reasonably well to the vertical velocity at a height of $1.37 \mathrm{Mm}$ (dashed line), just above the null point, which is located at $1.30 \mathrm{Mm}$. These two velocity components are in counterphase as a result of the bidirectional jet produced by the reconnection.

This nicely illustrates the fact that spectral lines are formed over a range of heights, rather than at one specific height, and that care must be taken when using them as probes of atmospheric conditions. But it also illustrates that such a multi-component signal could be used as evidence of reconnection via the characteristic bidirectional velocity pattern, as long as the reconnection happens in the height range where the line is formed. By contrast, in our simulation with null points in the transition region, we observe only the downflows because the line is primarily formed below the null points and reconnection region.

\subsection{Transition region null points, Hinode driver}

\subsubsection{General description}

In this simulation, we go back to the magnetic configuration of the first simulation studied (section 3.1), where the null points are located in the transition region. However, instead of using a localised, monochromatic piston driver, we use velocity data obtained with Hinode/SP (as described in the introduction) to drive the whole lower boundary. As before, we will not attempt to match the simulation to specific observations; the data simply serve as a more random and realistic velocity driver with a wider range of frequencies than our monochromatic piston.

\subsubsection{Synthesised limb observations}

Using the Hinode driver changes a number of things. In general, the velocity field in the atmosphere becomes much more complex. On the left-hand side, the first reconnection has its jet oriented vertically, as opposed to the horizontal orientation we get in the runs with a piston driver. This is a result of the different phase of the first waves to reach the null point. In this case, as the reconnection happens in the transition region, only the hotter material above the null point is ejected upwards, and there is little cool material above the null point to be accelerated by later reconnection events. As a result, only few and short cool jets are formed at all.

At the right-hand side null point, no significant reconnection happens until $130 \mathrm{~s}$, but then a quite major event occurs, with its jet oriented vertically. This leads to the ejection of a powerful hot jet, as seen in Figure 12. The jet is too hot to show up in Ca.

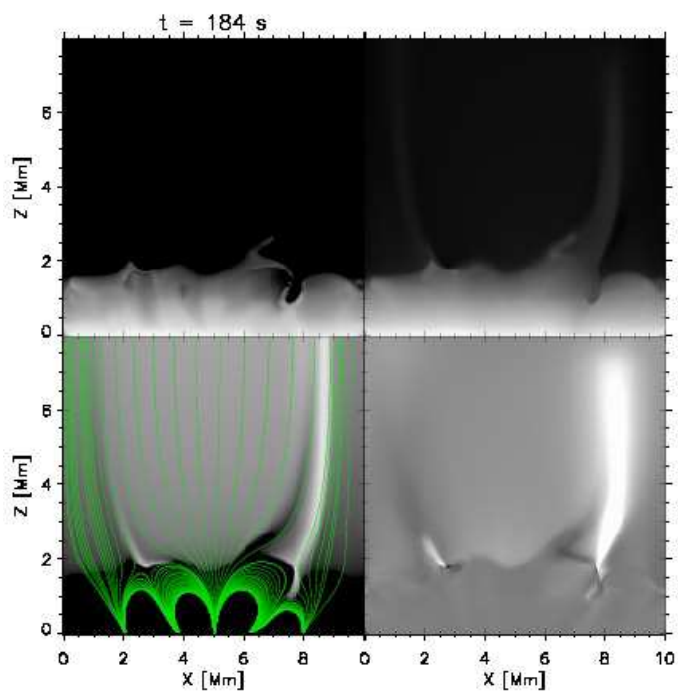

FIG. 12. - Plots of the Ca signal (top left), density (top right), temperature with magnetic field lines (bottom left), and fieldaligned velocity (bottom right) in the case with transition region null points and Hinode driver. The elapsed time is $184 \mathrm{~s}$. A strong and very fast hot jet is being produced by the reconnection at the right-hand null point. This figure is available as a movie in the electronic version of the Journal.

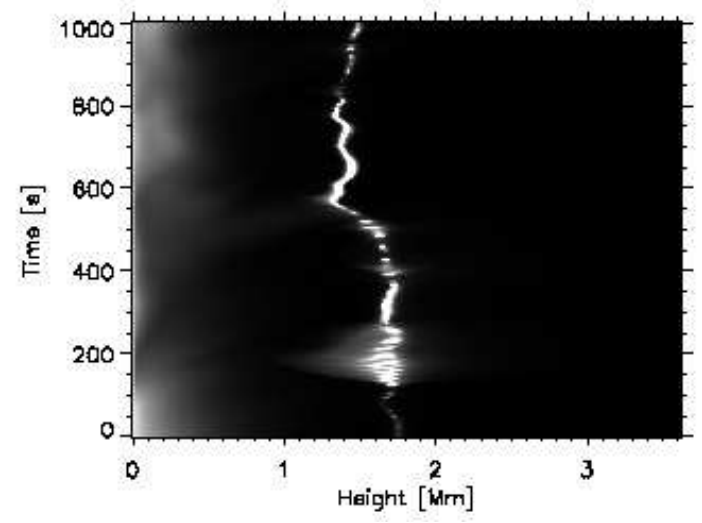

FIG. 13. - $\beta$ plot showing the height change of the right-hand null point with time when it starts out in the transition region and the Hinode driver is applied.

This latter reconnection event is actually quite longlasting, the flow direction only being reversed at $290 \mathrm{~s}$, and then only for $90 \mathrm{~s}$ before another $170 \mathrm{~s}$ period of vertically oriented reconnection. The sustained event releases quite a bit of energy from the magnetic field and changes its structure fairly significantly; among other things, the null point is moved down into the chromosphere by 3$400 \mathrm{~km}$ (Fig. 13). Later jets are fairly weak, the cool ones reaching only around $1 \mathrm{Mm}$ above the transition region (Fig. 14).

The long duration of the event is likely a result of the specifics of the driver. On the right-hand side of the box, the input velocity only rarely changes sign, leading to a fairly continuous flow in one direction, rather than the periodic reversals of the piston driver.

\subsubsection{Synthesised disk observations}




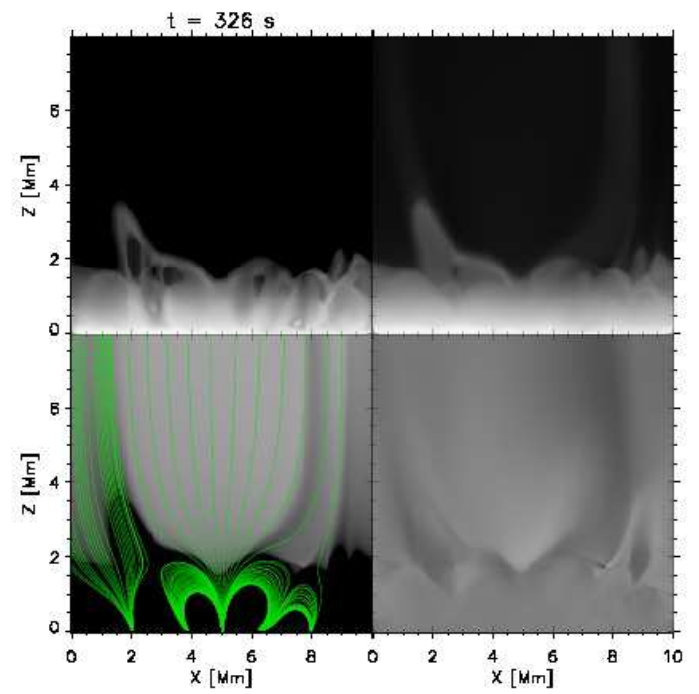

FIG. 14.- Plots of the Ca signal (top left), density (top right), temperature with magnetic field lines (bottom left), and fieldaligned velocity (bottom right) in the case with transition region null points and Hinode driver. The elapsed time is $326 \mathrm{~s}$. This figure shows the longest of the cool jets that appear in this simulation.
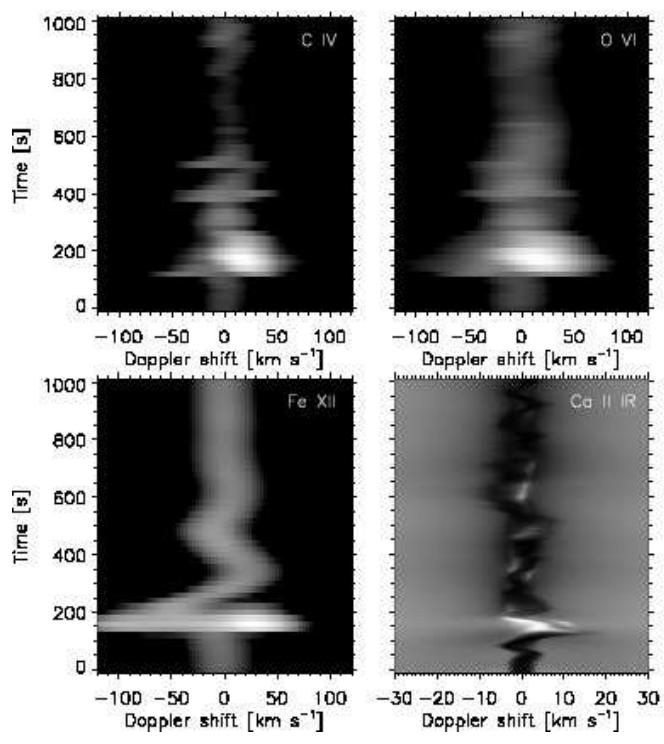

FIG. 15. - Spectra of the high null point Hinode driver case, centered at $x=7.6 \mathrm{Mm}$. The UV lines use logarithmic scaling and the $\mathrm{Ca}$ line uses linear scaling. The $\mathrm{C}$ and $\mathrm{O}$ lines show mainly redshifts because the matter moving upwards is heated out of their passbands. Fe shows not only the signal of the heated area around the reconnection point, but also of the fast hot jet to the side of it, shown in more detail in Fig. 16]

Because the null point in this simulation displays significant movement, both horizontally and vertically, and because the jets tend to follow the magnetic field, which is slightly inclined even in the lower corona, we can find interesting phenomena at several different locations when looking down as we would do if the events happened on the solar disk. We calculate the same $\mathrm{C}, \mathrm{O}, \mathrm{Fe}$, and $\mathrm{Ca}$ lines as above, and use the same spatial resolution and exposure times.

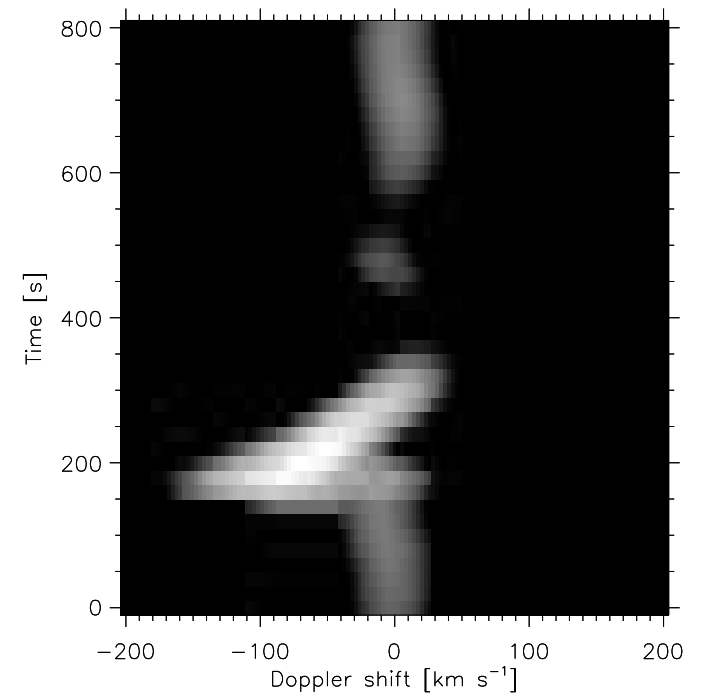

FIG. 16. - Fe XII spectrum of the high null point Hinode driver case, centered at $x=8.45 \mathrm{Mm}$ and using logarithmic scaling. This spectrum shows the very fast hot jet and its considerable blueshift of $160 \mathrm{~km} \mathrm{~s}^{-1}$.

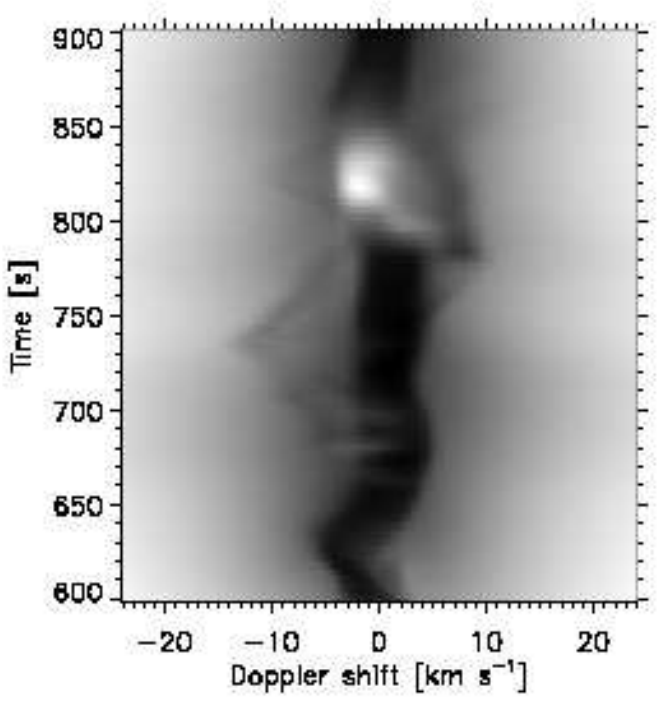

FIG. 17.- Ca II IR spectrum of the high null point Hinode driver case, centered at $x=7.9 \mathrm{Mm}$. Weak blueshifted excursions similar to those in Fig. 10 can be seen as a result of the null point having moved down after extended reconnection.

In Figure 15. we show $\lambda$-t plots of the different spectra centered at $x=7.6 \mathrm{Mm}$. This is the place where the transition region and coronal lines have their highest intensities, looking straight down on the reconnection region. The $\mathrm{C}$ IV line shows little bidirectionality in this case, having its maximum at redshifts of around $25 \mathrm{~km} \mathrm{~s}^{-1}$. The $\mathrm{O}$ vi line is bidirectional, but has much higher intensity in the red. The Fe XII line is more extended, but also has its maximum at the same redshift. This line not only includes emission from the region immediately surrounding the null point, but also from the upwards propagating fast hot jet between $x=8$ and $x=9 \mathrm{Mm}$. A spectrum centered at $x=8.45 \mathrm{Mm}$ showing this jet in more detail is shown in Figure 16. We can 
see the strong signature of the fast hot jet and its deceleration between 160 and $340 \mathrm{~s}$. It reaches an impressive blueshift of roughly $160 \mathrm{~km} \mathrm{~s}^{-1}$, matching the maximum field-aligned velocity from the simulation data.

The Ca II IR line shows only redshifts for this event (130-200 s), some of them rather powerful (up to $20 \mathrm{~km} \mathrm{~s}^{-1}$ ), and exhibits strong central reversal at around $140 \mathrm{~s}$, when the plasma is significantly heated by the reconnection. The later $\mathrm{Ca}$ spectrum is more complex, likely showing several overlapping velocity components as in the low null point piston driver case in section 3.2. as well as the effects of the movement of the null point itself. Of course, the velocity input from the Hinode driver includes a wide spectrum of frequencies rather than the single one of the piston, which serves to further complicate the simulated Ca spectrum.

The effects of having the null point move several hundred $\mathrm{km}$ downwards are visible in the Ca signal in some locations. In Figure [17, we show a portion of the spectrum at $x=7.9 \mathrm{Mm}$. Between 680 and $780 \mathrm{~s}$, there is a clear blueshifted excursion, similar to what we saw in the spectra of the simulations with chromospheric null points (Figs. 10 and 11). By the time of this event, the right-hand null point in this simulation is at a height of 1.4 Mm (Fig. 13), very close to the height of the null points in the other simulation.

\subsection{Chromospheric null points, Hinode driver}

\subsubsection{General description}

In this simulation, we again use the magnetic configuration that puts the magnetic null points in the chromosphere, but use the same Hinode driver as in the previous section. Once again this leads to extensive reconnection near the right-hand null point, but because that is now embedded in chromospheric plasma, it produces a major cool jet rather than the hot one produced when the null points are in the transition region. Again the reconnection changes the field and pushes the null point lower in the atmosphere after about $500 \mathrm{~s}$ (Fig. 18). Later jets are fairly weak and do not extend far above the transition region; neither do the jets produced by the weaker events at the left null point. Some jets are also produced by shock waves propagating from the lower boundary, especially in the magnetic channel connecting to $x=2 \mathrm{Mm}$ - the shock fronts are clearly visible in movies of the field-aligned velocity. This process is similar to the one that produces dynamic fibrils (Hansteen et al. 2006), but the jets produced this way tend to reach heights of no more than $0.5 \mathrm{Mm}$ above the transition region in this simulation.

\subsubsection{Synthesised limb observations}

In Figure 19, we show the $\mathrm{Ca} \mathrm{H}$ signal, density, temperature and field-aligned velocity at 216 s elapsed time, when the region heated by the first powerful reconnection event has reached its maximum extent. We see that the upper part of that region follows the field lines upwards; the total length of the heated region is more than $2 \mathrm{Mm}$. We also see the high velocity of the jet. Note that the heated region gets too hot to show up in the Ca signal.

In Figure 20. we show the situation at $350 \mathrm{~s}$, when the cool jet has reached its maximum extent. As we see, it reaches a height of nearly $8 \mathrm{Mm}$ above the lower bound-

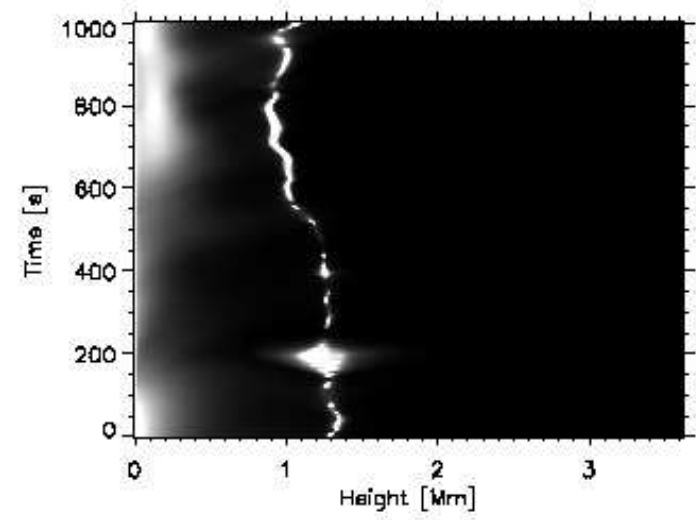

FIG. 18. - $\beta$ plot showing the height change of the right-hand null point with time when it starts out in the chromosphere and the Hinode driver is applied.

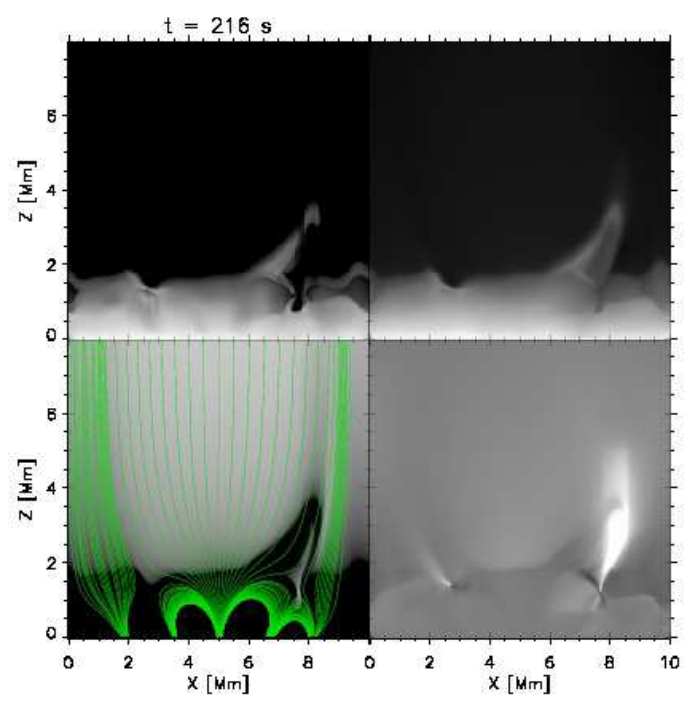

FIG. 19. - Plots of the Ca signal (top left), density (top right), temperature with magnetic field lines (bottom left), and fieldaligned velocity (bottom right) in the case with chromospheric null points and Hinode driver. The elapsed time is $216 \mathrm{~s}$. A powerful reconnection event is happening at the right-hand null point, heating a large area and propelling a cool jet upwards at great speed. This figure is available as a movie in the electronic version of the Journal.

ary, or $6 \mathrm{Mm}$ above the transition region. This cool jet shows up quite strongly in $\mathrm{Ca}$, and has a noticeable density enhancement compared to the surrounding corona. The velocity, as one would expect, is close to zero at this time.

This jet does not form a perfect parabola in a $z$ - $t$ diagram (it ascends slightly faster than it descends), but the best parabolic fit, using the method of De Pontieu et al. (2007a) and Heggland et al. (2007), yields a maximum velocity $v_{\max }$ of $52 \mathrm{~km} \mathrm{~s}^{-1}$, a deceleration $d$ of $250 \mathrm{~m} \mathrm{~s}^{-2}$, and a duration $P$ of roughly $430 \mathrm{~s}$. Although these values fall outside the range studied by Heggland et al. (2007) in their study of shock wave-driven dynamic fibrils - in particular, the maximum velocity is much higher than would reasonably develop through steepening of acoustic waves alone - they are a pretty good fit to the formula 


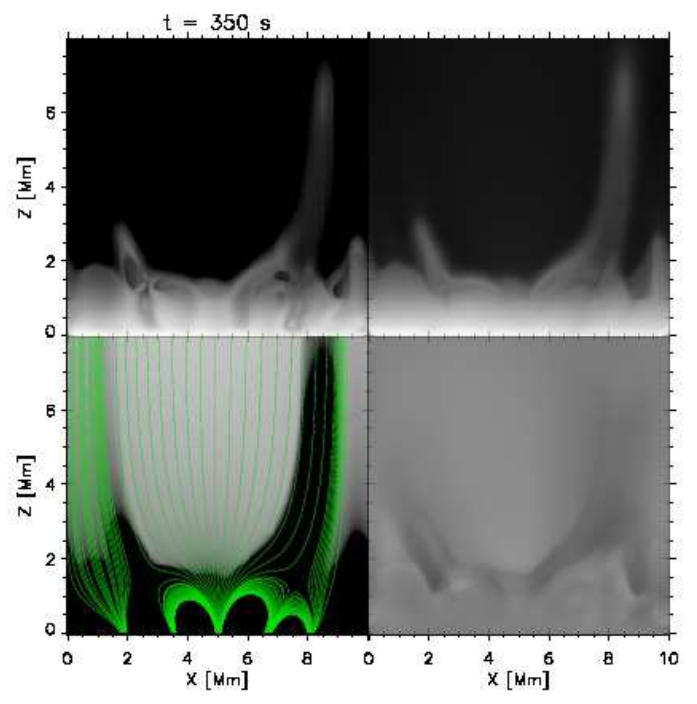

FIG. 20.- Plots of the Ca signal (top left), density (top right), temperature with magnetic field lines (bottom left), and fieldaligned velocity (bottom right) in the case with chromospheric null points and Hinode driver. The elapsed time is $350 \mathrm{~s}$. The cool jet has reached its maximum extent, $8 \mathrm{Mm}$ above the photosphere.

predicted and found in that paper, namely

$$
d=\frac{v_{\max }}{P / 2}
$$

not too surprisingly since the jet is driven by a shock, albeit one generated by reconnection rather than directly by photospheric motions.

\subsubsection{Synthesised disk observations}

In Figure 21] we show the simulated C IV, O VI, Fe XII, and Ca II IR spectra looking down on $x=7.65 \mathrm{Mm}$. As in the other cases, the TR lines are plotted at $650 \mathrm{~km}$ and $20 \mathrm{~s}$ resolution, and the $\mathrm{Ca}$ spectrum at $150 \mathrm{~km}$ and $2 \mathrm{~s}$.

$\mathrm{C}, \mathrm{O}$, and $\mathrm{Fe}$ all have huge maxima in connection with the first reconnection event, centered at 180-200 s - even with a lower intensity cutoff of $10^{-4}$ times the maximum intensity, as in all the UV spectral plots, only Fe shows any part of the background signal. The event shows up as a clear bidirectional jet in all three, with $50-70 \mathrm{~km} \mathrm{~s}^{-1}$ velocities; even up to $90 \mathrm{~km} \mathrm{~s}^{-1}$ in $\mathrm{O}$ VI. $\mathrm{C}$ and $\mathrm{O}$ have some of the same asymmetry as in the TR null point piston driver case (section 3.1), with slightly higher blueshifts than redshifts and slightly redshifted intensity maxima.

In $\mathrm{Ca}$, we get strong emission and a redshift of $17 \mathrm{~km} \mathrm{~s}^{-1}$ for this event. Later on, the spectrum gets more complex, as usual with the Hinode driver. However, it is notable that after the null point moves down at around $500 \mathrm{~s}$, the Ca spectrum mainly shows blueshifts, since the primary formation height is now above the reconnection region. This makes a nice contrast with the mainly redshifted spectrum of the high null point piston driver case (Fig. 7), where the null point is located almost $900 \mathrm{~km}$ higher up.

\subsection{Energy release estimates}

In Figure 22, we have plotted the energy release rate in the form of joule heating $\left(j^{2} / \sigma\right.$, where $j$ is the current

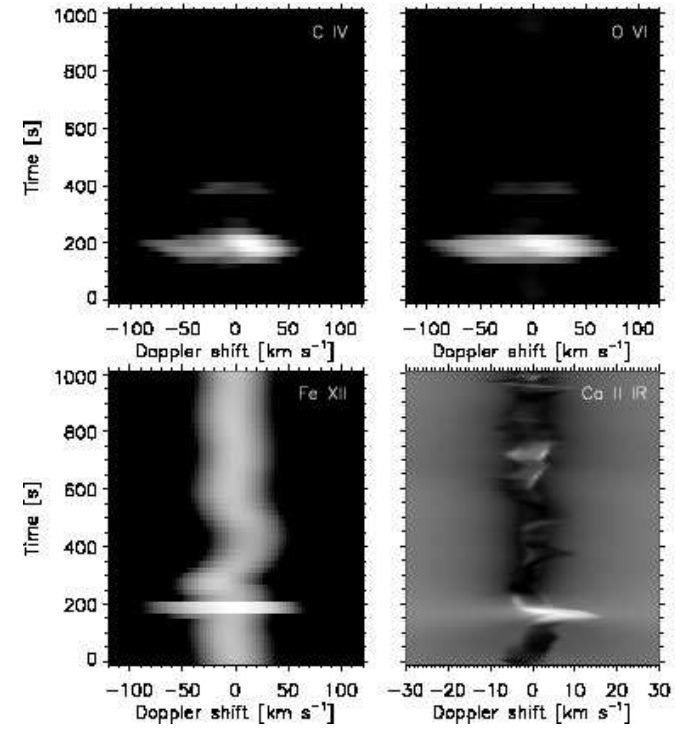

FIG. 21. - Spectra of the chromospheric null point Hinode driver case, centered at $x=7.65 \mathrm{Mm}$. The UV lines use logarithmic scaling and the $\mathrm{Ca}$ line linear scaling. All lines have huge maxima when the first major reconnection event happens. Fe is largely symmetric while the $\mathrm{C}$ and $\mathrm{O}$ lines have asymmetries similar to those in Fig. 7] Ca, formed at the lowest height, is redshifted, but the later signal is shifted more towards the blue after the null point moves to lower heights.

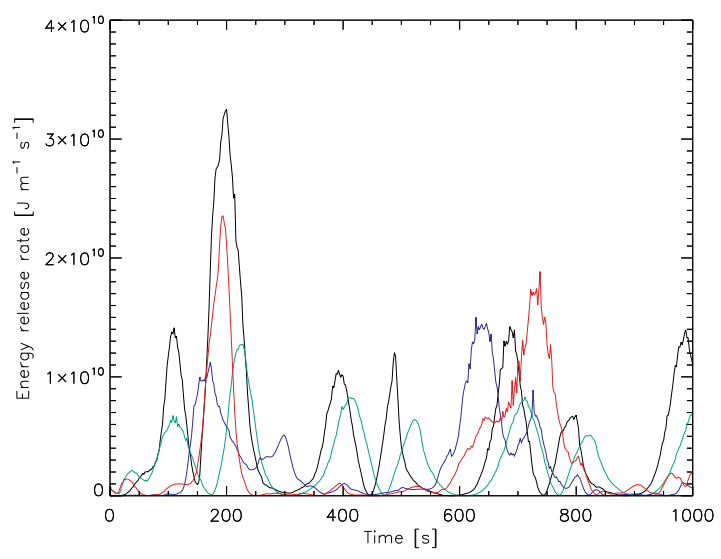

FIG. 22. - Energy release rate in the form of joule heating in the four different cases: transition region null point with piston driver (black), chromospheric null point with piston driver (green), transition region null point with Hinode driver (blue), and chromospheric null point with Hinode driver (red).

density and $\sigma$ the conductivity) in our four simulations. The energy release is calculated over the reconnection region, in this case defined as the region around the null point where the joule heating is above $5 \%$ of the value at the null point. The results are not very sensitive to the particular value of the threshold, and changing it from $1 \%$ to $10 \%$ changes the calculated energy release by less than $10 \%$. In the piston driver cases, we include the region around the left-hand null point, while we use the right-hand one in the Hinode driver cases.

As we would expect from the side views and spectra, the greatest peaks in the energy release occur in the transition region null point piston driver case (black line) 
TABLE 1

ENERGY RELEASE PER RECONNECTION EVENT effect (Wilhelm 2000, Fig. 8) may be happening at times in our model; see for example the left centre panel of Figure 3. where the mass flow is perpendicular to the field Tines and chromospheric matter is propelled upwards by

\begin{tabular}{lcccc}
\hline \hline & \multicolumn{2}{c}{ First event } & \multicolumn{2}{c}{ Strongest later event } \\
\multicolumn{1}{c}{ Case } & Energy [J] & Duration [s] & Energy [J] & Duration [ \\
\hline TR np/piston & $1.23 \times 10^{18}$ & 280 & $5.40 \times 10^{17}$ & 280 \\
Ch. np/piston & $5.48 \times 10^{17}$ & 280 & $4.19 \times 10^{17}$ & 280 \\
TR np/Hinode & $5.01 \times 10^{17}$ & 240 & $7.15 \times 10^{17}$ & 260 \\
Ch. np/Hinode & $3.31 \times 10^{17}$ & 100 & $7.18 \times 10^{17}$ & 280
\end{tabular}

and the chromospheric null point Hinode driver case (red line). The two piston driver cases are more regular, with double peaks (horizontal, then vertical jets) appearing every $300 \mathrm{~s}$. The Hinode driver cases have second peaks appearing between 600 and $800 \mathrm{~s}$, and the energy release is actually greater in these events than in the first one at around $200 \mathrm{~s}$, but the later events show only weak observational signatures. This is because the jets of these events are horizontally oriented, giving no Doppler shifts in the simulated spectra and not very tall jets, and also because the energy release happens over a longer period of time than at the first peak, at least in the case with chromospheric null points.

The energy release rate is given in $\mathrm{J} \mathrm{m}^{-1} \mathrm{~s}^{-1}$ because the total energy release will be dependent on the size of the reconnection region in the third dimension. In order to calculate the total energy release, we must assume an extent, and this value will necessarily be somewhat arbitrary. A value of $0.5 \mathrm{Mm}$ has been assumed in Table 1. The released energy per event is comparable to the generally assumed values for nanoflares, though not all the energy released in these events will be in the form of joule heating.

\section{DISCUSSION AND SUMMARY}

These simulations have been able to produce a number of jet phenomena through the same basic mechanism of wave-induced, or in the Hinode driver cases also convective flow-induced, magnetic reconnection. In particular, they appear to be the first 2D simulations to show the formation of spicule-like jets as a result of reconnection, while including most important physics; the main omission is time-dependent ionisation. The simulated spicules match observed lifetimes and lengths.

In this model of spicule formation, we also expect a brightening and broadening of UV lines at the footpoint of the spicule at the start of its lifetime; the heated plasma causing this is in general cooled down on a shorter timescale than the lifetime of the spicule. This UV bright point is a property our model shares with most earlier models that assume a sudden energy/pressure deposition as the source of spicules, rather than a velocity perturbation at photospheric heights. Although UV bright points are frequently observed in connection with spicules, some observations (e.g. Suematsu et al. 1995) indicate that this brightening happens after the ascending phase of the spicule, rather than at the beginning. This timing problem is not resolved by our model.

The UV brightenings that are produced in the reconnection regions have many of the same properties as observed explosive events, which could point towards a possible connection between spicules and explosive events, as suggested by Wilhelm (2000). One of the plasma acceleration mechanisms suggested in that paper, a slingshot several hundred $\mathrm{km}$. On the other hand, flow along the field proves much more effective in lifting chromospheric material in our simulations (right centre panel of Fig. 3 and lower two panels of Fig. 4); different field geometries could give different results.

Although our UV brightenings match many of the properties of explosive events, there are also some differences. Our events are generally very bright, with maximum intensities 50-100 times higher than the "quiet" profiles - in some cases, even 1000 times. Although some explosive events have strong brightenings, the majority are not particularly bright - Innes (2001) quotes typical brightening factors of 2-5. Also, we get significant signal in the lower coronal Fe XII $195 \AA$ A line, while explosive events rarely show up in coronal lines. Erdélvi et al. (1999) show one example of an explosive event brightening in TRACE $171 \AA$, but since it does not show up in the lower temperature $\mathrm{Mg}$ x $625 \AA$ line, they interpret it as a brightening of a transition region $\mathrm{O}$ vI line within the passband rather than the coronal $\mathrm{Fe} x$ line.

In one of our cases (Fig. 10), we also find only very weak Fe XII emission. Since the heating is quite strongly localised to the reconnection region, it becomes a blob of coronal plasma surrounded by low-temperature chromospheric material. In the low-temperature region, neutral hydrogen is present in significant proportions, and this leads to strong Lyman absorption of all lines with shorter wavelengths than $912 \AA$. This, combined with the heating events themselves being relatively weak, explains the lack of Fe emission in this case, and could also do the same for observed explosive events if in fact they occur relatively deep in the chromosphere. However, the $\mathrm{C}$ and $\mathrm{O}$ lines will be unaffected by this absorption, and are still stronger than observed. Also, the high velocities observed in many explosive events indicate a quite high formation height. A more likely explanation for the discrepancy may therefore be that our reconnection events are simply more energetic than those responsible for most explosive events. The temperatures reached are high enough $(>1 \mathrm{MK})$ that they might even have an $\mathrm{X}$-ray signal.

It should be noted that even the very strong first event in the TR null point piston driver case is completely absorbed by the neutral hydrogen above it. Therefore, the absence of observed signal in high-temperature lines does not necessarily mean that the plasma is not heated to such temperatures, as the emission could be absorbed by overlying cool matter.

Finally, wave-induced reconnection offers a natural explanation for the repetitive behaviour sometimes observed in explosive events (e.g. Chae et al. 1998; Ning et al. 2004). If these events are caused by reconnection induced by waves, we would naturally expect repetition at time scales of 3-5 minutes, corresponding to the dominant periods in the solar chromosphere and photosphere.

Although we find a number of similarities between our simulation results and observations, the complexity of the signals in our synthetic spectra and images shows 
that it is not surprising that the many different jet-like phenomena that are observed in the solar atmosphere have been, and remain to some extent, such a puzzle. For example, in the same simulations, with similar magnetic field geometry and driver, we find at different times jets that show significant chromospheric signal without transition region or coronal counterparts, jets that show only blueshifts in the chromosphere, jets that only show redshifts in the chromosphere, jets that do not show chromospheric signatures but are dominated by bidirectional flows in the transition region, and jets in the transition region lines that do not show any coronal counterpart. This is a natural consequence of the complex mix of magnetic field geometry, the history of the plasma motions, the narrow height/temperature range in which the observables are formed, and the varying mix of heating and acceleration in reconnection events. As a result, establishing the relationship between various types of events in the solar atmosphere is fraught with difficulties that can only be resolved by statistical comparisons of spatiotemporal data of high quality with advanced radiative MHD models.

This work was supported by the Research Council of Norway through grants 159483/V30, 170926, and 170935, as well as a grant of computing time from the Program for Supercomputing. B. D. P. was supported by NASA grants NNG06GG79G, NNX08AL22G, and NNM07AA01C (Hinode). L. H. thanks Øystein Langangen and Mats Carlsson for useful discussions.

\section{REFERENCES}

Beckers, J. M. 1968, Sol. Phys., 3, 367

Carlsson, M. 1986, Uppsala Astronomical Observatory Reports, 33

Chae, J., Wang, H., Goode, P. R., Fludra, A., \& Schühle, U. 2000, ApJ, 528, L119

Chae, J., Wang, H., Lee, C.-Y., Goode, P. R., \& Schühle, U. 1998, ApJ, 497, L109

Chen, P. F. \& Priest, E. R. 2006, Sol. Phys., 238, 313

De Pontieu, B., Hansteen, V. H., Rouppe van der Voort, L., van Noort, M., \& Carlsson, M. 2007a, ApJ, 655, 624

De Pontieu, B., McIntosh, S., Hansteen, V. H., Carlsson, M. Schrijver, C. J., Tarbell, T. D., Title, A. M., Shine, R. A., Suematsu, Y., Tsuneta, S., Katsukawa, Y., Ichimoto, K., Shimizu, T., \& Nagata, S. 2007b, PASJ, 59, 655

Dere, K. P. 1994, Advances in Space Research, 14, 13

Doyle, J. G., Popescu, M. D., \& Taroyan, Y. 2006, A\&A, 446, 327

Erdélyi, R., De Pontieu, B., \& Sarro, L. M. 1999, in ESA Special Publication, Vol. 448, Magnetic Fields and Solar Processes, ed. A. Wilson, 1345

Grossmann-Doerth, U. \& Schmidt, W. 1992, A\&A, 264, 236

Hansteen, V. H. 2005, in IAU Symposium 223, Multi-Wavelength Investigations of Solar Activity, ed. A. V. Stepanov, E. E. Benevolenskaya, \& A. G. Kosovichev, 385

Hansteen, V. H., De Pontieu, B., Rouppe van der Voort, L., van Noort, M., \& Carlsson, M. 2006, ApJ, 647, L73

Heggland, L., De Pontieu, B., \& Hansteen, V. H. 2007, ApJ, 666, 1277

Innes, D. E. 2001, A\&A, 378, 1067

Innes, D. E., Inhester, B., Axford, W. I., \& Wilhelm, K. 1997, Nature, 386, 811

Innes, D. E. \& Tóth, G. 1999, Sol. Phys., 185, 127

Karpen, J. T., Antiochos, S. K., \& DeVore, C. R. 1995, ApJ, 450, 422
Katsukawa, Y., Berger, T. E., Ichimoto, K., Lites, B. W., Nagata, S., Shimizu, T., Shine, R. A., Suematsu, Y., Tarbell, T. D.,

Title, A. M., \& Tsuneta, S. 2007, Science, 318, 1594

Madjarska, M. S. \& Doyle, J. G. 2003, A\&A, 403, 731

Ning, Z., Innes, D. E., \& Solanki, S. K. 2004, A\&A, 419, 1141

Osterbrock, D. E. 1961, ApJ, 134, 347

Roussev, I., Doyle, J. G., Galsgaard, K., \& Erdélyi, R. 2001a, A\&A, 380, 719

Roussev, I., Galsgaard, K., Erdélyi, R., \& Doyle, J. G. 2001b, A\&A, 370, 298

-. 2001c, A\&A, 375, 228

Sarro, L. M., Erdélyi, R., Doyle, J. G., \& Pérez, M. E. 1999, A\&A, 351, 721

Shibata, K., Nakamura, T., Matsumoto, T., Otsuji, K., Okamoto, T. J., Nishizuka, N., Kawate, T., Watanabe, H., Nagata, S., UeNo, S., Kitai, R., Nozawa, S., Tsuneta, S., Suematsu, Y., Ichimoto, K., Shimizu, T., Katsukawa, Y., Tarbell, T. D., Berger, T. E., Lites, B. W., Shine, R. A., \& Title, A. M. 2007, Science, 318, 1591

Shibata, K., Nishikawa, T., Kitai, R., \& Suematsu, Y. 1982, Sol. Phys., 77, 121

Sterling, A. C. 2000, Sol. Phys., 196, 79

Sterling, A. C., Shibata, K., \& Mariska, J. T. 1993, ApJ, 407, 778

Suematsu, Y., Wang, H., \& Zirin, H. 1995, ApJ, 450, 411

Takeuchi, A. \& Shibata, K. 2001a, ApJ, 546, L73

—. 2001b, Earth, Planets and Space, 53, 605

Tsiropoula, G., Alissandrakis, C. E., \& Schmieder, B. 1994, A\&A, 290,285

Vernazza, J. E., Avrett, E. H., \& Loeser, R. 1981, ApJS, 45, 635

Wilhelm, K. 2000, A\&A, 360, 351 